
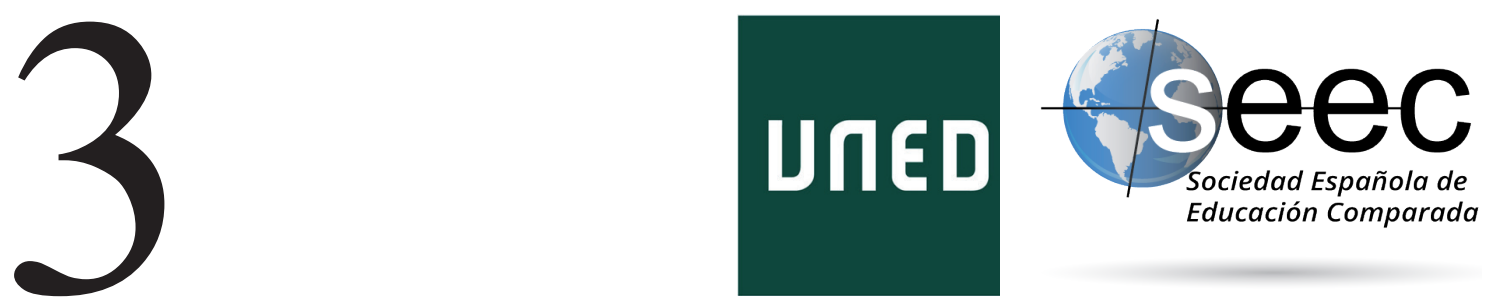

\title{
Evolución del caso japonés como referente internacional en la educación para la reducción del riesgo de desastres
}

Evolution of the Japanese case as an international reference in education for disaster risk reduction

\section{Elisa Gavari Starkie*; José Pastrana Huguet**}

DOI: $10.5944 /$ reec.32.2018.22319

\section{Recibido: 10 de julio de 2018 Aceptado: 4 de diciembre de 2018}

\footnotetext{
*Elisa Gavari Starkie: Profesora Titular de Educación Comparada en la UNED. Entre sus líneas de investigación destacan la Educación en otros Sistemas Educativos y las Prácticas Profesionales. Ha sido miembro de la red de formación de investigadores de la Comisión Europea a través de los Programas Intensivos. Ha publicado diversos manuales sobre las Prácticas Profesionales y ha impartido cursos prácticos de escritura en diversos formatos. Datos de contacto: E-mail: egavari@edu.uned.es

*José Pastrana Huguet. Graduado en Educación Social y con los siguientes títulos de Máster: En Protección Civil y Gestión de Emergencias; Sistemas Integrados de Gestión; Dirección y Gestión de Recursos Humanos; y Prevención de Riesgos Laborales. Es experto en Gestión y Dirección de Seguridad. Actualmente, forma parte del equipo investigador del proyecto en la UNED «Estudio comparativo de la educación para la prevención de los riesgos en desastres en sistemas educativos de Japón, Chile, Brasil y España». Trabaja en el Centro de Gestión de Emergencias Municipal de la Policía Local del Ayuntamiento de Ciutadella de Menorca. Doctorando en el programa de Doctorado en Educación de la Escuela Internacional de Doctorado de la UNED. Datos de contacto: E-mail: joseppastrana@msn.com
} 


\title{
Resumen
}

Este artículo ofrece el análisis de la educación para la reducción del riesgo de desastres en Japón. La selección de este país responde a su consideración como referente internacional tras las tres Conferencias Mundiales celebradas en su territorio sobre la reducción del riesgo de desastres (Yokohama 1994, Hyogo 2005 y Sendai 2015). La metodología del artículo descansa el estudio histórico del cambio conceptual en el concepto de reducción de desastres. Si bien en un principio simplemente se adoptaban medidas para paliar los desastres, en la actualidad, Japón ofrece un modelo integrado que aborda la gestión de los desastres. El texto ofrece la descripción del modelo japonés partiendo de la base legal adoptada en 1961 que regula la participación de todos los niveles administrativos en la materia con funciones muy definidas en un sistema integrado. El modelo japonés se completa con la introducción en el currículo escolar de dos estrategias clave como son la introducción en las asignaturas troncales de contenidos sobre gestión de riesgo de desastres, y el horario específico de estudio integrado diseñado por los docentes. De hecho, los docentes son actores fundamentales en la reducción de desastres naturales no solamente por la formación que recibe sino por su vinculación con la comunidad. En este sentido al artículo recoge la labor desde la sociedad civil, en particular a través de las asociaciones de voluntarios, que en ocasiones supera la labor del Ministerio de Educación. Se cierra el artículo con unas breves conclusiones sobre el modelo japonés.

Palabras clave: Japón; Reducción para el Riesgo de Desastres; Educación; Asociaciones de voluntarios; Formación docente

\begin{abstract}
This article offers the analysis of education for disaster risk reduction in Japan. This country has been selected as it is considered to be the reference on education for risk reduction. In fact the Three World Conferences in Disaster Risk Reduction (Yokohama 1994, Hyogo 2005 and Sendai 2015) have taken place in the Japanese territory. The methodology of the article lays on the historical study of the conceptual change of education for risk reduction. At the first stage only some measures in order to palliate disasters were adopted, at present, Japan offers an integrated model that tackles the risk management. The text offers a description of the Japanese model study starting from the 1961 Law that regulates the participation of all administrative levels with defined functions in the integrated system. The Japanese model also has introduced in the curriculum two key strategies: the introduction of contents referred to risk reduction management in main subjects and a specific timetable for integrated study designed by the teachers. In fact teachers are considered to be key actors in the disaster risk reduction not only for the training they receive but also for the commitment with the community. In this sense the article refers to the work of the civil society, in particular through the volunteer association, that many times surpass the work of the Ministry of Education. The article ends with some brief conclusions concerning the Japanese model.
\end{abstract}

Key Words: Japan; Disaster Risk Reduction; Education; Volunteer Associations; Teacher training 


\section{Introducción}

En el año 2018 se celebra la conmemoración de los 150 años de Relaciones Internacionales entre España y Japón. Este artículo pretende enmarcarse en este marco señalando la necesidad de revisar una de las mayores aportaciones de Japón en la educación internacional, como es, la prevención y reducción del riesgo de desastres. De hecho, la Representante Especial del Secretario General de las Naciones Unidas para la Reducción del Riesgo de Desastres es una japonesa ${ }^{1}$.

La Asamblea General de Naciones Unidas declaró a finales de los años 8o, la Década Internacional para la Reducción de los Desastres Naturales 1990-1999, al objeto de disminuir las pérdidas humanas y los daños materiales causados por los desastres. Este marco constituyó la base para la celebración de tres Conferencias Mundiales de las Naciones Unidas para la reducción del riesgo de desastres, que se han celebrado en el territorio japonés.

La primera Conferencia Mundial sobre Reducción del Riesgo de Desastres se celebró en Yokohama en 1994. Tuvo como resultado la aprobación de la Estrategia y el Plan de Acción para un Mundo más Seguro. Dicho texto proponía, entre otras cuestiones, un mayor énfasis en las ciencias sociales en campos como la investigación en el desarrollo de políticas públicas y aplicaciones prácticas, destacando los vínculos entre la reducción del riesgo de desastres y el desarrollo sostenible (EIRD², 1999). Además, la Estrategia y el Plan de Acción de Yokohama establecía un grupo de directrices para actuar en la prevención, preparación y mitigación del riesgo de desastres basadas en un grupo de principios que destacaban la importancia de la evaluación del riesgo, la preparación, la capacidad de prevenir, de reducir y mitigar desastres y la alerta temprana (IISD ${ }^{3}, 2015$ ).

En 1999 la Asamblea General de las Naciones Unidas adoptóla Estrategia Internacional para la Reducción de los Desastres para promover una «cultura de prevención». En este nuevo marco, en 2005 se celebró en Hyogo la Segunda Conferencia Mundial sobre la Reducción de Riesgos de los Desastres Naturales, que estableció un nuevo plan de acción para el periodo 2005-2015. Este plan considera la educación la clave para el desarrollo de una cultura de prevención de desastres. En este sentido se planteó la necesidad de usar el conocimiento, la innovación y la educación para construir una cultura de prevención y resiliencia en todos los niveles ${ }^{4}$.

La Tercera Conferencia Mundial se celebró en 2015 en Sendai. Se aprobó un nuevo marco de acción a quince años cambiando el énfasis de la gestión del riesgo al de desastres. El debate se materializó en el «Marco de Sendai para la Reducción del Riesgo de Desastres 2015-2030» e identifica siete metas mundiales para el 2030. Las primeras cuatro medidas proponen la reducción de los daños y pérdidas: de la mortalidad mundial producida por los desastres, del número de personas afectadas, las pérdidas económicas directas en relación con el producto interno bruto (PIB) mundial y los daños a las infraestructuras vitales e interrupción de los servicios básicos. Las tres siguientes medidas contemplan el aumento del número de países con estrategias nacionales y locales para

$1 \quad$ La Sra. Mami Mizutori fue nombrada Subsecretaria General y Representante Especial del Secretario General para la Reducción del Riesgo de Desastres, Oficina de las Naciones Unidas para la Reducción del Riesgo de Desastres (UNISDR) el 1 de marzo de 2018.

2 Estrategia Internacional para la Reducción de desastres.

3 International Institute for Sustainable Development.

4 Acción 3 del Marco de Acción de Hyogo 2005-2015. 
la reducción del riesgo de desastres, una mayor cooperación internacional con los países en desarrollo, y aumentar el acceso a la información, los sistemas de alerta temprana y las evaluaciones del riesgo de desastres ${ }^{5}$. Además, establece cuatro prioridades de acción enfocadas a comprender el riesgo de desastres, fortalecer la gobernanza del riesgo de desastres para gestionar el riesgo, invertir en la reducción del riesgo de desastres para la resiliencia y aumentar la preparación para casos de desastre a fin de dar una respuesta

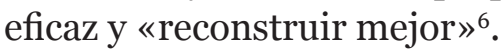

El destacado papel de Japón en el discurso internacional sobrela educación se completa con la Agencia Japonesa de Cooperación Internacional (JICA). A través de esta Agencia se realizan programas de formación, intercambio y tecnología, así como prestamos y subvenciones a fondo perdido para la reducción, construcción y rehabilitación con países en vías de desarrollo. La JICA coordina la cooperación con 90 oficinas alrededor del mundo que asisten a más de 100 países.

El presente artículo comienza mencionando algunas iniciativas abordadas a lo largo de la historia japonesa como las medidas en la construcción de edificios y la organización de la administración en reducción de riesgos de desastres en Japón. Si bien la arquitectura japonesa es especialmente sensible a adoptar estructuras y medidas para la prevención ante desastres, en este artículo nos focalizamos en las medidas educativas.

En concreto el presente texto comienza señalando la importancia de la organización administrativa en tres niveles para el diseño de los planes educativos en los diferentes niveles. En este marco el sistema educativo japonés considera a las escuelas como un agente clave vinculándose a las necesidades locales e incorporando dos estrategias básicas dentro de un modelo denominado integrado en el que se desarrollan dos modalidades fundamentales. Además, las asociaciones civiles tienen un papel clave en la prevención a lo que se añaden otros agentes. En este sentido el texto continúa señalando el papel primordial de los recursos humanos especialmente los docentes que reciben formación específica en la temática, y las sociedades civiles. El artículo concluye con unas breves reflexiones acerca del modelo japonés con el fin de ilustrar posibles estrategias para la inclusión de una asignatura de con contenidos asociados a la preparación ante distintos riesgos o su inclusión transversal en las asignaturas troncales.

Se espera que este artículo sirva para una revisión del caso español en el que se está considerando la inclusión de una asignatura con contenidos para una educación en la preparación para la gestión del riesgo o su inclusión transversal en las asignaturas troncales. Hasta ahora La Ley Orgánica para la Mejora de la Calidad Educativa (LOMCE) y los correspondientes reales decretos incluyen elementos transversales relacionados con la seguridad, la protección civil y el desarrollo de actitudes de responsabilidad con el medio ambiente ${ }^{7}$. No obstante, es necesario que se refuercen las estrategias educativas, así como que se amplíe la responsabilidad a la sociedad civil.

5 II. Resultado previsto y objetivos, punto 18, Marco de Sendai para la Reducción del Riesgo de Desastres 2015-2030.

6 IV. Prioridades de acción, punto 20 a 34, Marco de Sendai para la Reducción del Riesgo de Desastres 2015-2030.

7 Real Decreto 126/2014, de 28 de febrero, por el que se establece el currículo básico de la Educación Primaria.

Real Decreto 1105/2014, de 26 de diciembre, por el que se establece el currículo básico de la Educación Secundaria Obligatoria y del Bachillerato. 


\section{La organización administrativa japonesa}

La ubicación geográfica de Japón le convierte en uno de los países del mundo más sensibles a los fenómenos geológicos y climáticos por lo que esta condición le ha obligado a adoptar medidas y acciones para protegerse de las amenazas de los desastres. A lo largo de la historia la cultura japonesa de reducción del riesgo de desastres comenzó en la arquitectura adoptando estructuras en las construcciones de los edificios que les permitieran evitar los desastres de los terremotos. En el año 607 durante período Asuka, se construyó en Nara el Templo Hōryū-ji (Templo de la Ley Floreciente). Esta estructura, una Pagoda de cinco pisos construida por el Príncipe Shōtoku, tiene características arquitectónicas únicas a prueba de terremotos, y constituye todavía hoy, una de las construcciones de madera más antiguas en el mundo. A esto se añade en el siglo XVI la acción del jefe del clan, Shingen Takeda que, en el período feudal, construyó un dique para evitar inundaciones conocido como Shingen tsutsumi, donde convergen los ríos Midai y Kamanashi en la ciudad de Kai, en la Prefectura Yamanashi.

$\mathrm{Si}$ bien la arquitectura japonesa es especialmente significativa a nivel internacional por las estructuras y formas arquitectónicas que ayudan a prevenir el riesgo de los desastres, hay que señalar que Japón ha diseñado una estrategia nacional que permite que participen en la reducción de riesgos todos los niveles administrativos. La primera normativa adoptada en la prevención de desastres se aprobó durante la restauración Meiji, en pleno proceso de modernización. En 1880 se aprobaba la «Ley de Provisión y Ahorro para Desastres Naturales ${ }^{8}$, orientada a la administración de los recursos necesarios para afrontar los desastres en el caso de producirse. A esto se añadió la creación de la primera comisión dedicada a hacer frente a los desastres naturales (Penuel y Statler, 2011).

Tras los desastres de la Segunda Guerra Mundial el gobierno definió una estrategia nacional que perdura hasta nuestros días en la reducción de desastres. La inflexión en la administración japonesa tiene mucho que ver con el paso del tifón de Ise-wan en 1959 que causó numerosos daños y que despertó un movimiento social. La presión al gobierno permitió el aumento en la inversión de recursos para la mitigación y prevención de desastres naturales, y la aprobación de la Ley Básica de Medidas contra Desastres en 1961 que constituye el marco legislativo base vigente, que, aunque ha sido reformada constituye todavía hoy la base para la administración de desastres en Japón. Esta Ley se completa con leyes específicas como la Ley de control de inundaciones de 1949 (modificada en 2001 y 2013); la Ley de prevención de deslizamientos de 1958 o la Ley de medidas especiales para volcanes activos de 1978 .

El texto de la Ley Básica de Medidas contra Desastres de 1961 define las jurisdicciones y responsabilidades en la administración de desastres en tres niveles en un sistema integrado. Cada nivel se encarga del diseño y ejecución del plan para la prevención, la mitigación y preparación, la respuesta de emergencia y recuperación y la rehabilitación. Los tres niveles con las correspondientes funciones son las siguientes:

- El Gobierno central y las corporaciones públicas designadas a través de la Oficina del Gabinete del Consejo Central de Gestión de Desastres ${ }^{9}$ se encarga de deliberar sobre cuestiones relacionadas con la gestión de desastres de conformidad con las solicitudes del Primer Ministro o Ministro de Estado para la Gestión de Desastres, y de la promoción de medidas integrales para la gestión

8 Director General de Administración de Desastres, Oficina de Gabinete, Japón.

9 Cabinet office. 
de desastres. El Ministro de Estado para la Gestión de Desastres es el responsable de la coordinación de las políticas y medidas en gestión de desastres, contando con la asistencia del Director General de la Oficina de Gestión de Desastres de la Oficina del Gabinete y su departamento. El Gabinete envía un informe anual al Parlamento del estado de la gestión de desastres y especifica las necesidades presupuestarias en la gestión de desastres para su debate.

- A nivel prefectural el Gobernador es el encargado de la formulación y ejecución del plan de administración de desastres y su coordinación Integral. Al Consejo de Gestión de Desastres Prefectural le corresponde la elaboración del Plan Local de Gestión de Desastres.

- A nivel municipal la gestión de desastres se realiza en virtud de un Plan local, elaborado por el Consejo Municipal de Gestión de Desastres, el jefe de la entidad local y el gobierno municipal. Todas las medidas especificadas en el Plan local se implementan en cooperación con la policía, los cuerpos de bomberos, los grupos comunitarios, las escuelas y otras partes interesadas. El Gobierno japonés actúa como promotor en la prevención y reducción de desastres.

Por último, cabe señalar la importancia del reconocimiento por parte del gobierno japonés de los eventos relacionados con la prevención en la reducción de riesgos que se publicitan en los sitios web del Gobierno sobre leyes y políticas relacionadas con los desastres, material educativo e información sobre eventos. En 1960 el gobierno japonés declaró el 1 de septiembre como el «Día de Administración de Desastres» Bosai ${ }^{10}$ no Hi en conmemoración del Terremoto de Kanto del año 1923 en el que murieron más 100.000 personas en Tokio. Otra celebración señalada es el reconocimiento de la «Semana de la Administración de Desastres» Bosai Shukan que se celebra en agosto, coincidiendo con la temporada de tifones. En esta semana se realizan prácticas que tienen como objetivo preparar a la ciudadanía ante todo tipo de calamidades naturales.

Otras celebraciones son la declaración de 17 de enero como el «Día de Voluntarios y Reducción de Desastres», la semana del 15 al 21 de enero como Semana de los Voluntarios y Reducción de Desastres». Durante estas jornadas, los gobiernos a nivel nacional, prefectural y municipal ofrecen eventos de sensibilización y simulacros prácticos de capacitación. En todo el país se organizan ejercicios y maniobras para las brigadas de bomberos, residentes locales, empresas y otros colectivos sociales.

\section{La prevención de riesgos de desastres en el currículo escolar japonés}

Tras la Segunda Guerra Mundial se aprobó la Ley de Educación Escolar en el año 1947, que establecía «el cultivo de hábitos necesarios para una vida sana, segura y feliz y el desarrollo armonioso de la mente y el cuerpo ${ }^{11}$ como uno de los objetivos de la educación

10 La palabra "BOSAI" viene de dos vocablos japoneses. El primero, "BO”, tendría varias traducciones al castellano: protección, prevención, mitigación, rehabilitación o reconstrucción. El segundo, "SAI", se puede traducir como «desastre». Por lo tanto "BOSAI" es mucho más que mitigación o prevención de desastres, mucho más amplio, es un concepto que va más allá y ve la globalidad de los fenómenos, se trata de una gestión de los riesgos. 
escolar. El texto de la ley se completó con la aprobación de la Ley de Salud Escolar en 1958, que amplió la política de educación sanitaria incluyendo las áreas de salud escolar y almuerzos escolares. La aportación fundamental consistió en un nuevo enfoque en el que se vinculaba la salud de los niños a la seguridad en las escuelas. Según Shiwaku y Shaw (2016) con anterioridad al Gran Terremoto Hanshin-Awaji del año 1995, los principales contenidos en educación para el riesgo de desastres en Japón se centraban en la realización de ejercicios de evacuación en caso de desastres.

El Ministerio de Educación (MEXT) dio un paso más en el año 2006, y definió una estrategia básica para promover el compromiso nacional para reducir los riesgos de desastres. Esta nueva Ley involucraba a las instituciones educativas en todos los niveles que debían desarrollar la educación sobre Reducción del Riesgo de Desastres en su localidad de acuerdo con las especificidades de sus comunidades y de los riesgos de desastre (riesgos volcánicos, de inundaciones, terremotos, tsunamis...). Esta ley se completó con la Ley de Salud Escolar, que se revisó el 18 de junio de 2006, y el resultado fue el cambio en la denominación por la de Ley de Seguridad y Salud Escolar. Esto significó que el peso de la seguridad en la ley se situó a un nivel similar al de salud. Como comenta Kitagawa (2016) está ley está regulada dentro de un marco de políticas de múltiples riesgos llamado «Seguridad Escolar» organizada en tres ámbitos: la seguridad general de la vida cotidiana de los estudiantes, la seguridad vial y la reducción de desastres.

Como ocurre con la Ley Básica de Medidas contra Desastres, la educación para la reducción de desastres es una competencia compartida entre el gobierno, las 47 prefecturas y el ámbito municipal. El marco de este programa de educación se establece en el Plan Básico de Gestión de Desastres, que, a su vez, se divide en planes regionales, prefecturales y municipales. Este modelo organizativo permite que la educación para desastres pueda realizarse teniendo en cuenta los riesgos específicos de cada comunidad y las necesidades locales, variando en función de los riesgos a los que deban enfrentarse. En este nuevo marco legal cada escuela, siguiendo las directrices ministeriales, diseña e implementa un Plan de Seguridad Escolar con el que se obliga a implantar iniciativas de seguridad adecuadas. Este plan, contempla las actividades formativas que debe recibir el alumnado relacionadas con la prevención y actuación en caso de desastres, establecen protocolos, convenios y áreas de responsabilidades en situaciones de emergencia.

Japón constituye un ejemplo internacional en la integración de la reducción en riesgos de desastres en el currículo escolar. El mismo Ministerio de Educación (MEXT) establece en los planes de 2002, las directrices educativas. La incorporación de la prevención en el currículo de manera transversal supone incluir el contenido en las asignaturas troncales de Ciencias Sociales, Ciencias Naturales, Geografía o Educación Física. En cada asignatura se incluyen temáticas relacionadas con la prevención del riesgo para desastres específicos de la disciplina y orientados al conocimiento, y además se desarrollan las habilidades de observación e investigación.

A esta estrategia se añade una segunda modalidad denominada "período de estudio integrado» y consiste en que se anima a la escuela y al maestro a utilizar el plan de estudios de manera creativa ofreciendo una oferta interdisciplinaria e integral. En este espacio curricular se incluye, entre otros, la comprensión internacional y educación ambiental y de bienestar. Los temas de prevención en riesgos de desastres tratan preferentemente temas específicos de la localidad de residencia. El énfasis es mayor en regiones afectadas recientemente por desastres naturales o en áreas de alto riesgo. Se realizan diversas actividades como simulacros de evacuación, manejo de equipos de extinción 
de incendios, visitas a parques de bomberos, visitas a zonas afectadas, etc. Por ejemplo, como mencionan Selby y Kagawa, (2012) utilizando 50 períodos de clase por año durante el período de estudio integrado, una escuela de primaria en la prefectura de Kochi realiza actividades como la creación de mapas de evacuación, espectáculos de marionetas sobre prevención en riesgos de desastres para niños más pequeños, simulacros de evacuación y práctica de habilidades en primeros auxilios.

\section{Tabla 1}

Síntesis a modo de ejemplo de actividades para la reducción del riesgo de desastres en el currículo escolar legislado por MEXT

NIVEL

PRIMARIA

(edad: 7-12)

\section{CURRÍCULUM}

Educación para la Vida ( $2{ }^{\circ}$ grado): instalaciones públicas y trabajadores, por ejemplo, visita a un parque de bomberos y la profesión de bombero.

Ciencias ( $5^{\circ}$ grado): trabajo de agua potable.

Ciencias ( $6^{\circ}$ grado): cambio de la orografía influenciada por erupciones volcánicas, terremotos e inundaciones.

Salud y educación física ( $5^{\circ}$ grado): Prevención de lesiones

Economía doméstica ( $5{ }^{\circ}$ y $6^{\circ}$ grado): ejercicios de cocina al aire libre.

\section{SECUNDARIA DE \\ PRIMER CICLO}

(edad: 13-15)
Ciencias: características de las erupciones volcánicas y los terremotos.

Salud y educación física: prevención de lesiones y primeros auxilios en desastres.

Economía doméstica: ejercicios de cocina al aire libre.
SECUNDARIA DE SEGUNDO CICLO (edad: 16-18)
Salud y educación física: entrenamiento de primeros auxilios en tiempos de desastre.

Ética: dignidad para la vida, relación con la naturaleza y la ciencia.

Ciencias: Mecanismo de erupciones volcánicas y terremotos.

Economía doméstica: cocinar y vivir en tiempos de desastre.

Fuente: Nakamura, 2007 EDM-NIED ${ }^{12}$. 
Tabla 2

Síntesis que recoge las actividades en educación sobre el riesgo de desastres en la escuela (legislado por MEXT)

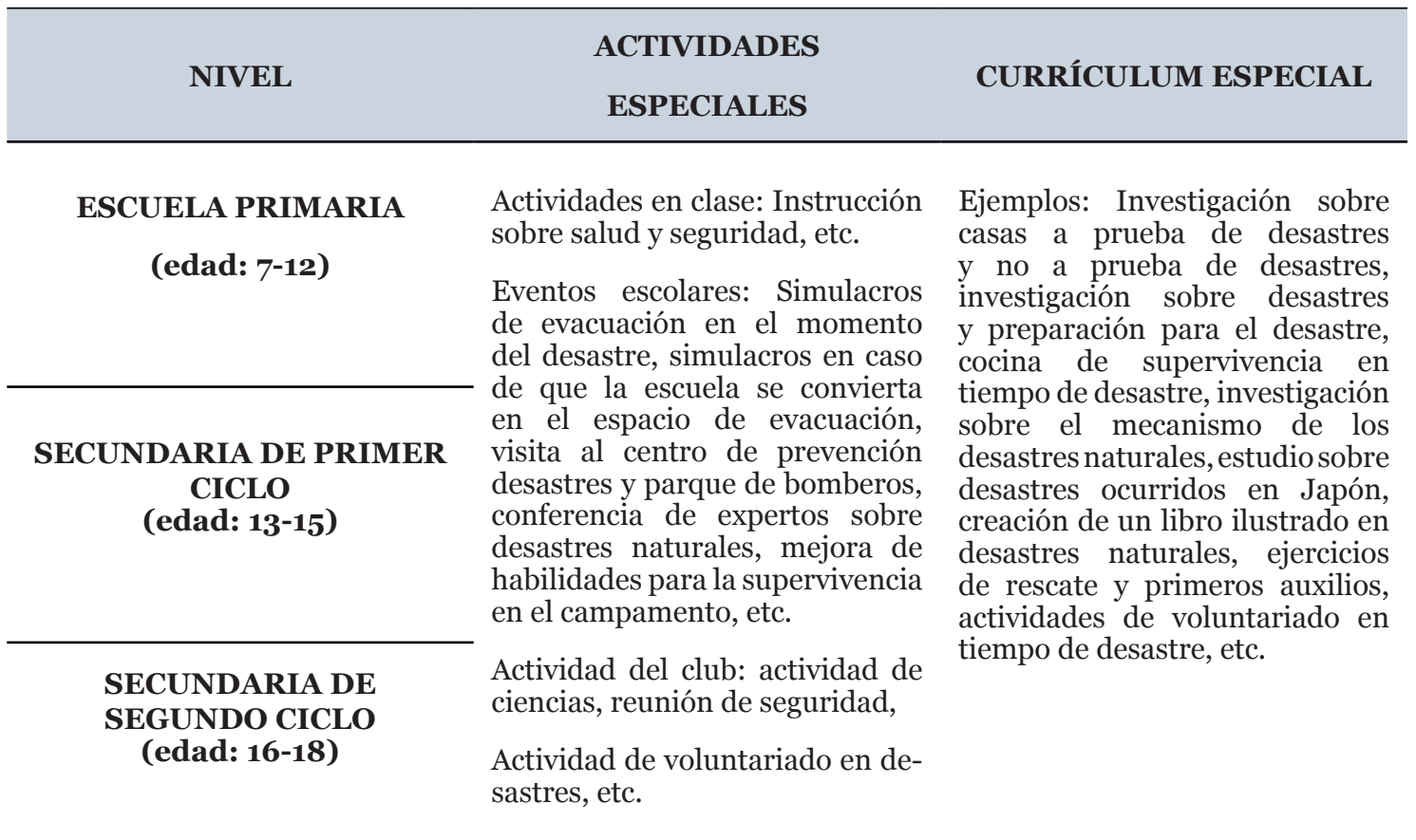

Fuente: Nakamura, 2007 EDM-NIED.

Tras el gran terremoto de Japón Oriental se ha revisado el currículo con el fin de dar un nuevo enfoque que consiste en el fomento de actitudes proactivas en el marco de la gestión de riegos. Lo que se pretende es que los alumnos se conviertan en agentes activos para contribuir a los esfuerzos de reconstrucción después de un desastre. Sobre este nuevo enfoque el Ministerio de Educación, Cultura, Deportes Ciencia y Tecnología (MEXT) elaboró un plan quinquenal a implementar entre los años 2012 y 2016 sobre la promoción de la seguridad escolar, basado en la Ley de Seguridad y Salud Escolar. En este plan se incluía la implementación integral y efectiva de las medidas de seguridad escolar administradas por cada escuela. Con ello se pretendía fortalecer las medidas de seguridad en desastres, incluyendo la protección de los hogares, el transporte y la educación en prevención de desastres, así como la necesidad de promover una seguridad escolar basada en la cooperación coordinada entre los hogares y la comunidad local. El plan propone cuatro medidas para incorporar la seguridad en las escuelas: - una protección escolar adecuada; - instalaciones escolares adecuadas; - el desarrollo de medidas sistemáticas de seguridad en las escuelas; y - el desarrollo de la seguridad escolar a través de la cooperación con los hogares y las comunidades.

\section{Variedad de agentes en la prevención}

A partir del Gran Terremoto de Hanshin-Awaji de 1995 la reducción del riesgo de desastres en el sector educativo comenzó a ser un tema de interés entre académicos que realizan estudios interdisciplinarios. Fruto de este interés se creó en el año 2000 la 
Asociación Japonesa de Educación en Seguridad ${ }^{13}$ que actualmente es una de las principales organizaciones académicas en la promoción de la educación sobre reducción del riesgo de desastres. A esta Asociación se añaden las instituciones nacionales de capacitación docente que han sido sometidas lo mismo que las universidades nacionales a diversas reformas. Una de las más importantes es la conversión de las universidades nacionales en entidades administrativas independientes en el año 2004. Estas reformas que se vinculaban al ámbito de la seguridad escolar incluían cambios en la renovación de la licencia docente y el establecimiento de escuelas de postgrado profesionales para la educación a nivel de Máster. Si bien no necesariamente consideraban la educación sobre reducción del riesgo de desastres como un tema vital.

En este sentido el desastre del terremoto de la costa del Pacífico en la región de Tōhoku del año 2011, y las proyecciones de que grandes terremotos afectarán el área metropolitana de Tokio y Tokai y Tonankai en el oeste de Japón han fomentado la colaboración entre las escuelas y la comunidad. Lo que ha significado que la educación para la reducción de desastres sea una necesidad para el personal docente de la escuela. Desde esta nueva perspectiva se pretende que los docentes y alumnos adquieran conocimientos científicos sobre desastres, así como primeros auxilios y habilidades de asesoramiento.

El desastre del año 2011 estimuló numerosas discusiones sobre políticas de seguridad escolar y capacitación destinadas a fortalecer las capacidades de los docentes para responder ante un desastre. Tras el informe del Consejo Central de Educación emitido el 21 de marzo de 2012, el Gabinete resolvió un plan de promoción de la seguridad escolar el 27 de abril de 2012, declarando que la educación en seguridad debería estar presente en los programas de capacitación docente. Las recomendaciones de este Consejo dirigido por académicos, diseñadores de políticas y profesionales, enfatizó la necesidad de programas formales de reducción del riesgo de desastres en dos niveles: Durante la formación de los nuevos docentes y para los educadores en activo que no recibieron educación especializada en reducción del riesgo de desastres durante su preparación académica. La capacitación en el servicio de los maestros existentes generalmente implica el uso de centros de capacitación prefecturales o municipales, que otorgan la formación legal requerida, nivel de gestión, entrenamiento en el trabajo y otras oportunidades de competencia.

En respuesta a estas necesidades de mejora de las competencias docentes un número creciente de centros de capacitación prefecturales o municipales están brindando programas de formación e investigación sobre reducción del riesgo de desastres. Por ejemplo, el mencionado por Selby y Kagawa (2012) en Tohoku en donde varios divulgadores fueron asignados al centro de capacitación, con el propósito de investigar y desarrollar materiales didácticos de reducción del riesgo de desastres a través del ordenador.

En Japón las escuelas son lugares que educan a los estudiantes sobre los desastres naturales y su prevención, pero, además constituyen espacios que mitigan los efectos de estos desastres proporcionando refugio y sirviendo como centros que ayudan a la recuperación, en las que se recibe y proporciona ayuda a la comunidad. Otro ejemplo como señala Selby y Kagawa (2012), es el del Departamento de Reducción del Riesgo de Desastres y el Consejo de educación en la ciudad de Kamaishi en la prefectura de Iwate que desarrollan materiales educativos sobre maremotos destinados a dotar a los estudiantes de capacidades para sobrevivir y ayudar a otros en caso de un tsunami. Se elaboró una guía del maestro donde se sugieren una serie de formas prácticas de insertar los riesgos de tsunami, terremoto y problemas de seguridad en todos los niveles de grado.

13 The Japanese Association of Safety Education. 
Takashi (2015) menciona las actividades realizadas en la Universidad de Miyagi ${ }^{14}$. En concreto, en la escuela profesional de posgrado se realizó un curso de educación de reducción del riesgo de desastres en octubre de 2014. En dicho programa se matricularon a unos 20 estudiantes de maestría de pregrado y docentes en servicio para aprender temas específicos sobre reducción del riesgo de desastres en las escuelas. Los estudiantes asistieron a conferencias conjuntas sobre políticas nacionales sobre seguridad escolar, desarrollo de marcos internacionales para la reducción del riesgo de desastres y la gestión de refugios de emergencia. Los científicos de un observatorio meteorológico local y bomberos impartieron conferencias especiales y talleres sobre los peligros naturales como los terremotos, tsunamis y tifones y respuestas de primeros auxilios, diseñados específicamente para la enseñanza basada en lecciones específicas aprendidas del desastre del Gran terremoto de Japón oriental del año 2011.

A nivel nacional el Ministerio de Educación (MEXT) se dedica principalmente a la formación de los supervisores y el personal de los consejos de educación prefectural, personal que a su vez pueda continuar con la capacitación de docentes en su propia localidad. La Oficina del Gabinete y el Ministerio de Educación (MEXT) han desarrollado y distribuido algunos materiales referencia para el maestro, así como otros educativos de apoyo para los estudiantes (como folletos o películas) para los diferentes niveles de grado. En el nivel local son las Juntas Locales de Educación las que deciden el peso específico que debe tener la reducción en riesgo de desastres en la capacitación docente.

\section{El rol del voluntariado en la gestión y prevención de desastres}

La sociedad japonesa se define por su visión tradicional de la comunidad. En palabras de Marta Pena de Matsushita (2012) podríamos definir la sociedad japonesa como una armonía jerarquizada denominada «Katarizada». Esta organización social se caracteriza por un fuerte sentimiento de pertenencia al grupo y de cooperación social por encima de las opciones individualistas, lo que ha propiciado la creación de las asociaciones de voluntarios. De hecho, los Chōnaikai, asociaciones comunitarias organizadas por los residentes locales realizan funciones tan diversas como actividades contra la delincuencia, campañas de seguridad vial, prevención de incendios y desastres, saneamiento, promoción de amistades mutuas, actividades culturales y recreativas, ayuda mutua, transmisión de información desde el ayuntamiento y representación de vecindarios ante los gobiernos locales.

Kitagawa (2016) señala que en la mayoría de los barrios de las ciudades y pueblos existen organizaciones voluntarias de prevención de desastres denominadas Jishu-bosaisoshiki o Jishubo abreviado. Estos términos se traducen literalmente por organización autónoma para la reducción de desastres. Dichas asociaciones las dirigen generalmente bomberos retirados y líderes comunitarios, que trabajan conjuntamente con las corporaciones municipales, y que realizan eventos de concienciación y simulacros de desastres para la comunidad. La actividad de las asociaciones supera en ocasiones la impulsada por las distintas instituciones gubernamentales. De hecho, a través de la Oficina del Gabinete del gobierno se apoya a las asociaciones mediante las siguientes acciones: 
- Proporcionar información de utilidad a los voluntarios para la realización de sus tareas voluntarias;

- Ofrecer instalaciones para facilitar reuniones entre el voluntariado donde se realizan actividades de intercambio de información y puntos de vista respecto a temas logísticos y de actuación;

- Facilitar asistencia a los gobiernos locales, proporcionando información y conocimiento adquirido en la gestión de desastres;

- Promover una amplia colaboración entre las actividades de los voluntarios cuando suceden los desastres.

Kitagawa (2016) señala dos categorías en la educación para la reducción de los desastres que se desarrollan en el ámbito formal, no formal e informal: la educación financiada con fondos públicos y la organizada por las asociaciones de voluntarios. Continúa el autor Kitagawa (idem) señalando que, tras los recortes presupuestarios del gobierno, la educación para desastres desarrollada por los voluntarios es más extensa que la financiada con fondos públicos. Como menciona Kitagawa (2016) lo interesante de la formación para desastres organizada desde el voluntariado es su naturaleza bidimensional. Una primera dimensión para la formación de los voluntarios se realiza a través de la capacitación formal e informal. A esto se añade una segunda dimensión en que la educación en desastres se adquiere a través de la misma participación en las actividades de voluntariado. Estas actividades propician el aprendizaje y la asimilación de las conductas necesarias para afrontar los desastres.

En los últimos años se ha añadido el consorcio sin ánimo de lucro e independiente llamado «Organizaciones Voluntarias de Japón Activas en Desastres» (JVOAD) y se encarga fundamentalmente de organizar la capacitación, el desarrollo de pautas para aclarar las funciones de los voluntarios, y la promoción de una cultura del voluntariado. Está compuesto por 23 organizaciones entre las que destacan: la Cruz Roja, Caritas, el Ejército de Salvación o el Consejo de Bienestar Social (Shakai Fukushi Kyogikai).

\section{Conclusiones}

La celebración de los 150 años de relaciones diplomáticas entre España y Japón en 2018 debe de servir para encontrar puntos de encuentro y e intercambio de propuestas educativas entre ambos países. Este artículo recoge un enfoque diferente en la educación para la reducción de riesgos. Si bien en España todavía no se ha abordado el tema con profundidad, el caso de Japón es un referente internacional por su modelo de prevención en el que el gobierno, las asociaciones civiles y las escuelas forman un sistema integrado. Además, Japón ha asumido el papel de liderazgo en la ONU organizando las tres Conferencias Mundiales en Reducción de Riesgos de Desastres en su territorio, y participando activamente en la elaboración de los Marcos de Acción que constituyen el marco internacional de actuación. Si en los años noventa el énfasis descansaba en el desarrollo sostenible como se recogió en la Conferencia de Yokohama a principios del siglo XXI se apostó por el desarrollo de la cultura de prevención como se recogió en Hyogo. En la actualidad el marco de Sendai ha definido las estrategias para la gestión del riego de desastres. La acción internacional se completa con las acciones de JICA que ofrece la 
transferencia de la experiencia japonesa y ofrece asistencia al resto de países a través de la formación, el intercambio de especialistas, exportación de tecnología y financiación a países menos desarrollados.

Si bien la primera normativa adoptada en la prevención de desastres se aprobó durante la restauración Meiji a partir de 1868, es tras el fin de la Segunda Guerra Mundial cuando se aprueba la Ley Básica de Medidas contra Desastres que, a pesar de haber sido reformada parcialmente, constituye todavía el marco legal de referencia. Esta ley establecía tres niveles integrados de la administración que desempeñaba cada uno funciones en la reducción de riesgos: el gobierno central, prefectural y local. Estas acciones administrativas se completan con las acciones desde las asociaciones civiles y las escuelas que participan activamente. La singularidad del estudio del caso japonés nos revela que la organización y gestión de los desastres es un sistema en el que la educación se destina a la prevención, mitigación y preparación en caso de desastres. Se trata de un sistema en el que las autoridades, comunidades e implicados trabajan de manera integrada para crear una comunidad de ciudadanos educados ante los desastres que conforman una sociedad resiliente.

Japón cuenta con uno de los modelos educativos más avanzados del mundo en la reducción del riesgo de desastres especialmente tras la aprobación de la ley de 2002 que incluye en el currículo escolar dos modalidades para el desarrollo la reducción de desastres. La primera se trata de incluir la educación para la prevención dentro del currículo de manera transversal. La segunda es la estrategia del denominado período integrado que concede al docente un destacable papel creativo, permitiéndole organizar las actividades junto con otros actores vinculados a la reducción de desastres.

Otra de las características que definen la educación para la reducción de desastres en Japón es que desde el año 2006 cuando se aprobó la Ley de Seguridad y Salud Escolar del año 2006 la prevención vincula a la salud en el marco de una flexibilización de las administraciones implicadas lo que permite la adaptación de las actividades formativas a los escolares atendiendo a las características locales.

Podemos decir que la educación para la prevención implica a varios actores no solamente vinculados a la escuela como, por ejemplo, la Asociación Japonesa de Educación en Seguridad. A esto se añade el trabajo de investigación en el que participan varias Universidades que han realizado investigaciones y han definido directrices para que los docentes adquieran las competencias adecuadas para formar parte de este sistema de gestión en el que la escuela ocupa un papel relevante, no únicamente en el ámbito educativo, sino también como refugio.

Tan importante como la educación desarrollada en prevención y reducción del riesgo de desastres en las escuelas es la desarrollada en el ámbito no formal en la que las asociaciones civiles desempeñan una labor fundamental en las comunidades para la reducción de riesgos, ofreciendo oportunidades de capacitación a toda la ciudadanía en estrecha colaboración con las instituciones gubernamentales que apoyan y promocionan la participación de la ciudadanía en estas asociaciones ofreciendo asesoramiento y recursos. Asimismo, las redes de asociaciones de voluntarios participan activamente en actividades educativas con la comunidad escolar configurándose como un elemento más del sistema educativo formal en la reducción del riesgo de desastres. Mediante este sistema toda la comunidad adquiere la capacitación adecuada y participa de manera en el caso de producirse un evento desastroso.

Para terminar, la educación para la reducción de los desastres se asienta en Japón en tres factores inexorablemente interrelacionados. En primer lugar, la ubicación geográfica 
de este país le ha convertido en uno de los países más sensibles a nivel global a los fenómenos geológicos y climáticos lo que le ha llevado a la necesidad de adoptar medidas para evitar y mitigar esos riesgos. En segundo lugar, las particularidades socioculturales del pueblo japonés vinculados al concepto de afrontar, adaptarse y reponerse a los desastres sufridos a lo largo de su historia desde una organización social jerarquizada en armonía en la que prima el grupo sobre el individuo. Por último, un fuerte sentimiento nacional y de deber en la cooperación ciudadana ante la adversidad, que ha sido promovido e implantado por el Estado en los últimos 300 años.

\section{Referencias bibliográficas}

Agencia de Cooperación Internacional del Japón (JICA), (2001). Cooperación de la JICA para la reducción del riesgo de desastres. Una sociedad para todos resiliente a los desastres. Integración de los retos para la reducción del riesgo de desastres en el desarrollo sostenible. Recuperado de: https://bit.ly/2tnzrJZ

Alexander, D. (2015). Disaster and Emergency Planning for Preparedness, Response, and Recovery. Oxford Research Encyclopedia of Natural Hazard Science. Recuperado de: https://goo.gl/oaQ7V2

Asamblea General de las Naciones Unidas (1999). Decenio Internacional para la Reducción de los Desastres Naturales: nuevas disposiciones (EIRD). Resolución A/54/497 de 1 de noviembre. Recuperado de: https://bit.ly/2MvWT3j

Cabinet Office, Government of Japan (2015). Implementation Handbook for Disaster Resilience Education at the Regional Level. Recuperado de: https://goo.gl/CyprZq

Cabinet Office, Government of Japan (2017). White Paper in Disaster Management in Japan 2017. Recuperado de: https://goo.gl/izSYmS

Dueñas, C. (2011). El cambio hacia una cultura de prevención. Conferencia virtual Iberoamericana febrero-marzo 2011. Recuperado de: https://goo.gl/mLv2Fm

España (2013). Ley Orgánica 8/2013, de 9 de diciembre, para la mejora de la calidad educativa. «BOE» núm. 295, de 10 de diciembre de 2013, páginas 97858 a 97921.

Gobierno de España (2015). Informe Nacional del Progreso en la Implementación del Marco de Acción de Hyogo (2013-2015) - Interim. Dirección General de Protección Civil y Emergencias. Recuperado de: https://goo.gl/YS4vNC

Government of Japan. Basic Act on Education (Act No. 120 of December 22, 2006). Recuperado de: https://bit.ly/2OVJ9A8

Government of Japan. Disaster Countermeasures Basic Act (Act No. 223 of 15 November 1961; revised June 1997). National Land Agency, Japan. Recuperado de: https:// bit.ly/2K5gcPE

Government of Japan (2015). Disaster Management in Japan. Director General for Disaster Management Cabinet Office, Government of Japan. Recuperado de: https://goo.gl/vgcbo7 
International Institute for Sustainable Development (2015). Boletín de Negociaciones de la Tierra (ENB). 26(15). Sábado, 21 de marzo de 2015. Recuperado de: http://enb. iisd.org/vol26/enb2615s.html

Japan Voluntary Organizations Active in Disaster (JVOAD). http://jvoad.jp

Kitagawa, K. (2016). Disaster preparedness, adaptive politics and lifelong learning: a case of Japan. International Journal of Lifelong Education, 35 (6), 629-647. doi:10 $.1080 / 02601370.2016 .1231230$

Kitagawa, K. (2016). Preparing for the worst: disaster education in Japan. East Asia Forum: Economics, Politics and Public Policy in East Asia and the Pacific. Recuperado de: https://goo.gl/oq8qXA

Ministry of Education, Culture, Sports, Science and Technology (MEXT), Government of Japan, (2012). Plan on the Promotion of School Safety (2012-2016). Recuperado de: https://goo.gl/2d3tWV

Nakamura, T. (2007). Disaster Risk Education at School in Japan. Earthquake Disaster Mitigation Research Center (NIED) (EDM). Recuperado de: https://goo.gl/ocgzWs

Naciones Unidas, Estrategia Internacional para la Reducción de Desastres, Naturales (EIRD), (1999) Un mundo más seguro en el siglo XXI: Reducción de Riesgos y Desastres Naturales. En: https://bit.ly/2QQh63b

Oficina de las Naciones Unidas para la Reducción del Riesgo de Desastres (UNISDR), (2005). Marco de Acción de Hyogo para 2005-2015: Aumento de la resilencia de las naciones. Conferencia Mundial sobre la Reducción de los Desastres, 2005, Kobe Hyogo, Japón. Recuperado de: https://bit.ly/Rqnqys

Oficina de las Naciones Unidas para la Reducción del Riesgo de Desastres (UNISDR), (2015). Marco de Sendai para la Reducción del Riesgo de Desastres 2015-203o. En: https://goo.gl/2Q4WVw

Oficina de las Naciones Unidas para la Reducción del Riesgo de Desastres (UNISDR). (2015). Memoria de la Tercera Conferencia Mundial de las Naciones Unidas sobre la Reducción de Riesgo de Desastres. Recuperado de: https://goo.gl/yVfeiY

Oficina de las Naciones Unidas para la Reducción del Riesgo de Desastres (UNISDR). (2007). Towards a culture of prevention: disaster risk reduction begins at school, good practices and lessons learned. Recuperado de: https://goo.gl/iDr9xV

Pena de Matsushita, M. (2011). La Cultura de Japón: Mitos, Educación, Identidad Nacional y Sociedad. Editorial Kaicron. ISBN: 978-987-1758-02-9.

Penuel, K. \& Statler, M. (2011). Encyclopedia of disaster relief (Vol. 2). SAGE.

Sawabi, O. (2012). Education and Disaster Reduction. The Japan Journal, Februari 2012. Recuperado de: https://bit.ly/2trRzlE

Secretaría Interinstitucional de la Estrategia Internacional para la Reducción de Desastres, Naciones Unidas (EIRD/ONU). (2004). Vivir con el riesgo: Informe mundial sobre iniciativas para la reducción de desastres. En: https://goo. $\mathrm{gl} / 9 \mathrm{Sn} 19 \mathrm{G}$ 
Selby, D., Kagawa, F. (2012). Disaster risk reduction in school curricula: case studies from thirty countries. United Nations Children's Fund (UNICEF); United Nations Educational, Scientific and Cultural Organization (UNESCO). En: https://goo.gl/ i14DH1

Shiwaku, K., Sakurai, A., \& Shaw, R. (2016). Disaster Resilience of Education Systems: Experiences from Japan. Springer Japan.

Takashi, O. (2015). Assisting the Recovery of School Education in Natural Disaster Emergencies: Roles of a Local Teacher Training University in Tohoku. Bulletin of Support Center for Revival in Education, Miyagi University of Education. En: https://goo.gl/6XZQUM

The World Bank (2016). Making Schools Resilient at Scale: The Case of Japan. En: https://goo.gl/oMbGPy

Unidad Regional para América Latina y el Caribe de las Naciones Unidas (1999). EIRD Informa-Revista para América Latina y el Caribe. Tema especial: Prevenir Recompensa. 14, 1999. En: https://bit.ly/2JYAUxq

Yasuyuki, K. Cipullo, L. (2012). Background Report Law and Regulation for the Reduction of Risk from Natural Disasters in Japan a National Law Desk. International Federation of Red Cross and Red Crescent Societies (IFRC) Survey. Recuperado de: https://goo.gl/QFzu2e 